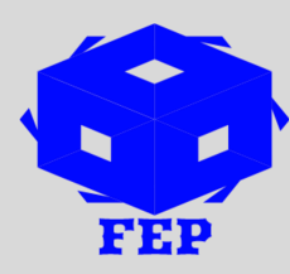

\title{
EXPERIMENTAL STUDY OF CONCRETE PERFORMANCE
}

Sang Kee ${ }^{1}$, Yuhee Park $^{2}$, Eun $\mathrm{Choi}^{3}$

${ }^{1,2,3}$ Department of Civil \& Environmental System Engineering,

Konkuk University, Seoul, Korea

*Corresponding Author: Sang Kee

Article Received: 09-12-19
Accepted: 02-03-20
Published: 27-03-20

Licensing Details: Author retains the right of this article. The article is distributed under the terms of the $\begin{array}{lllll}\text { Creative } & \text { Commons } & \text { Attribution-NonCommercial } & 4.0 & \text { License }\end{array}$ (http://www.creativecommons.org/licences/by-nc/4.0/) which permits non-commercial use, reproduction and distribution of the work without further permission provided the original work is attributed as specified on the Journal open access page

\section{ABSTRACT}

This study was experimental in nature and conducted with the view to make comparison between two samples. The first sample consisted of concrete with rice husk ash mixed in it and the other sample was without such addition. The first test conducted to test the performance was simple measurements. The results show that for the sample without addition of rice husk ash, the density was 2355.97 and for included sample, the density was 2354.44 with insignificant differences ( $t$ stat $=0.766, \mathrm{P}>.05)$. For $\mathrm{V}-\mathrm{B}$ test, the sample without addition of rice husk as was 8.34 and for include sample, it was 8.01. The differences for $\mathrm{V}-\mathrm{B}$ for both samples were statistically insignificant ( $\mathrm{t}$-stat=1.431, $\mathrm{P}>$.05). The slump test without for the sample without addition of rice husk was 12.75 and for included sample, it was 18.56. The difference was statistically significant (t-stat=2.455, $\mathrm{P}<.05)$. The compressive strength for excluded sample was 24.32 and for included sample was 20.01. The results were statistically insignificant ( $\mathrm{t}$-value $=1.13, \mathrm{P}>.05$ ). For flexural strength test, for excluded sample, the average score was 9.02 and for included sample, the average score was 9.19. The difference was statistically insignificant ( $\mathrm{t}$-stat=1.45, P>.05). Overall, the results lead to the conclusion that there are insignificant differences of addition of rice husk ash in concrete.

Keywords: Concrete, Experiment, Compressive Strength, Flexural Test.

\section{INTRODUCTION}

Concrete is important component of modern urban life. Because of different conditions, there is requirement of improving the strength of concrete by using various combination of materials. In this regard, a lot of researches are conducted to find out the optimum solutions. One component 
which was also researched and found to be providing greater strength to the concrete is the artificial fiber. These artificial fibers can consist of various combinations and improve concrete performance in terms of toughness, load bearing capacity after cracks, deformation capability, flexural impact, and general tensile strength. Thus, when artificial fiber is added to the concrete, its strength improves in terms of flexural toughness, ductility, tensile strength, and impact strength (Mohammadi, Singh, \& Kaushik, 2006). Steel fiber reinforced concrete which is a type of artificial fiber-based concrete is the focus of the study. The use of the Steel fiber reinforced concrete is increased in settings such as mining, pavements, precast tunneling, housing, flooring and industrial usage (yazici, Inan, \& Tabak, 2007). The characteristics of Steel fiber reinforced concrete can be changed by bringing variation in the concrete formulation and fiber material type including fiber concentration, fiber orientation, fiber distribution, fiber geometry, and so on (Zollo, 1997). Previous studies investigated the effects of concrete fiber combination on different qualities of concrete. For example, study by Eren and Celik (1997) investigated the steel fibers and silica fume effects on characteristics of high-strength concrete. The findings were that workability of concrete reduces as the proportion of fiber and fume is increased. In this study the objective is to investigate how addition of steel fiber influence the performance of the rice husk ash concrete performance in terms of modulus of elasticity, splitting tensile strength, flexural tensile strength and compressive strength.

\section{EXPERIMENT DESIGN}

The design of the study is experimental means we made two separate samples. One sample was consisted of addition of rice husk ash concrete, while, the other sample was consisted of without rice husk ash concrete. The performance of the both samples were tested using various tests. The materials used in the experiment are as follows;

The rice husk ash was used in the study which had specific gravity of 1.95 and bulk density of $0.835 \mathrm{~g} / \mathrm{cc}$.

The Portland cement with a pecific gravity of 4.12 is used in the study. The initial and final setting times of the cement was 135 and 210 minutes respectively.

Low carbon hooked end-based steel fiber was used in the experiment. It had length of $35 \mathrm{~mm}$ and diameter of $0.75 \mathrm{~mm}$, with density of $8.43 \mathrm{~g} / \mathrm{cm} 3$, and aspect ratio of 60 .

For maintenance of workability of fresh concrete, a commercial AC Green slump was used. It was kept constant in mass basis which was about $1 \%$ of binder content of concrete

For fine aggregate, we used the good quality river sand. The river sand had dry density of 1735 $\mathrm{kg} / \mathrm{m} 3$, specific gravity of 2.87 , and fineness modulus of 2.45 . The aggregate was passed from 20 $\mathrm{mm}$ and only $10 \mathrm{~mm}$ sieve were retained. The retained sieve had dry density of $1475 \mathrm{~kg} / \mathrm{m} 3$ and dry density of 3.2.

\section{Mixture Composition and Preparation}

The mixed design is made by M10 and 20 grade concrete. Water cement ratio were kept constant at 0.5 for M20 and 0.45 for M10; and binder content was kept constant for $415 \mathrm{~kg} / \mathrm{m} 3$ for M20 and $370 \mathrm{~kg} / \mathrm{m} 3$ for M10. Aggregate weight was assessed using the volume of aggregate. By bringing change in the Portland cement concrete, fresh concrete containing $2.5 \%$ to $20 \%$ rice 
husk were prepared. Fresh fiber reinforced concretes which consisted of various ratios of steel fiber in volume basis ranging from 0.25 to $1 \%$ were prepared. By introducing fiber or rice husk ash, aggregate weight for cubic meter was adjusted. The procedure of mixing the fiber-reinforced concrete starts with sand and gravel placement in dry mixed and concrete mixer for approximately 60 seconds. The mixing water was continuously mixed for about 120 seconds. The remaining plasticizer and mixing water are added and mixed. The composition was cast into specific mold and vibrated for removing any trapped air. Next, 24 hours were waited for mold to settle down and for demolding.

\section{Testing Method}

The fiber reinforced rice husk ash concrete qualities were investigated in this experimental study. For experiment, slump cone was used and the tests included compressive strength, flexural strength and splitting tensile strength. For experiment purpose, two separate samples were designed. One set of sample labelled as Sample 1 had rice husk ash added in to concrete; while, for sample 2 was the label for second sample which was without the rice husk ash added in to it. For both samples, a total of 36 sub-samples were collected at different time intervals in order to get the reliable results.

\section{RESULTS}

Results of the study are as follows;

Table 1: Unit Weight and Workability

\begin{tabular}{|c|c|c|c|c|c|c|}
\hline & \multicolumn{3}{|c|}{ Without Rice Husk Ash } & \multicolumn{3}{c|}{ With Rice Husk Ash } \\
\cline { 2 - 7 } No. & $\begin{array}{c}\text { Sample 1-Density } \\
\text { Kg/M3 }\end{array}$ & $\begin{array}{c}\text { Sample } \\
1-\text {-V-B }\end{array}$ & $\begin{array}{c}\text { Sample 1-Slump } \\
(\mathrm{cm})\end{array}$ & $\begin{array}{c}\text { Sample 2- } \\
\text { Density Kg/M3 }\end{array}$ & $\begin{array}{c}\text { Sample } \\
2 \text {-V-B }\end{array}$ & $\begin{array}{c}\text { Sample 2- } \\
\text { Slump (cm) }\end{array}$ \\
\hline S1 & 2488 & 6.5 & 17 & 2590 & 7.5 & 22 \\
\hline S2 & 2434 & 6.8 & 16 & 2576 & 7.3 & 21 \\
\hline S3 & 2490 & 6.1 & 15 & 2574 & 8.2 & 20 \\
\hline S4 & 2467 & 6.9 & 18 & 2543 & 8.4 & 20 \\
\hline S5 & 2456 & 7.3 & 19 & 2578 & 8.5 & 18 \\
\hline S6 & 2400 & 7.4 & 20 & 2400 & 8.7 & 19 \\
\hline S7 & 2461 & 7.9 & 21 & 2412 & 7.9 & 19 \\
\hline S8 & 2491 & 8.1 & 20 & 2389 & 9.1 & 18 \\
\hline S9 & 2455 & 8.2 & 17 & 2376 & 8.3 & 17 \\
\hline S10 & 2412 & 7.4 & 16 & 2365 & 8.5 & 17 \\
\hline S11 & 2387 & 7.5 & 17 & 2345 & 8.5 & 17 \\
\hline S12 & 2367 & 7.5 & 14 & 2367 & 8.4 & 18 \\
\hline S13 & 2345 & 8.2 & 13 & 2387 & 8.3 & 18 \\
\hline S14 & 2456 & 8.7 & 12 & 2390 & 7.6 & 18 \\
\hline S15 & 2378 & 8.4 & 11 & 2387 & 8.4 & 19 \\
\hline S16 & 2390 & 8.9 & 12 & 2376 & 8.9 & 19 \\
\hline S17 & 2320 & 9.1 & 12 & 2365 & 8.4 & 19 \\
\hline S18 & 2318 & 9.2 & 11 & 2354 & 7.5 & 18 \\
\hline S19 & 2356 & 9.4 & 10 & 2367 & 7.3 & 17 \\
\hline
\end{tabular}




\begin{tabular}{|l|l|l|l|l|l|l|} 
S20 & 2347 & 9.4 & 8 & 2390 & 7.2 & 16 \\
\hline S21 & 2336 & 9.3 & 9 & 2365 & 7.3 & 19 \\
\hline S22 & 2338 & 9.9 & 9 & 2345 & 7.1 & 18 \\
\hline S23 & 2391 & 9.8 & 8 & 2377 & 8.3 & 17 \\
\hline S24 & 2286 & 9.1 & 8 & 2387 & 8.8 & 19 \\
\hline S25 & 2267 & 9.4 & 8 & 2398 & 8.9 & 19 \\
\hline S26 & 2265 & 9.2 & 7 & 2309 & 8.4 & 17 \\
\hline S27 & 2256 & 9.4 & 9 & 2312 & 8.2 & 15 \\
\hline S28 & 2311 & 9.1 & 12 & 2132 & 7.4 & 16 \\
\hline S29 & 2269 & 8.9 & 11 & 2345 & 7.8 & 17 \\
\hline S30 & 2255 & 8.7 & 11 & 2310 & 7.5 & 17 \\
\hline S31 & 2234 & 8.4 & 12 & 2333 & 7.9 & 19 \\
\hline S32 & 2294 & 8.2 & 13 & 2332 & 7.5 & 20 \\
\hline S33 & 2245 & 8.1 & 11 & 2311 & 7.2 & 22 \\
\hline S34 & 2276 & 8.5 & 10 & 2289 & 7.3 & 22 \\
\hline S35 & 2278 & 7.9 & 10 & 2290 & 7.7 & 21 \\
\hline S36 & 2298 & 7.4 & 12 & 2294 & 8.3 & 20 \\
\hline Ave & $\mathbf{2 3 5 5 . 9 7}$ & $\mathbf{8 . 3 4}$ & $\mathbf{1 2 . 7 5}$ & $\mathbf{2 3 5 4 . 4 4}$ & $\mathbf{8 . 0 1}$ & $\mathbf{1 8 . 5 6}$ \\
\hline
\end{tabular}

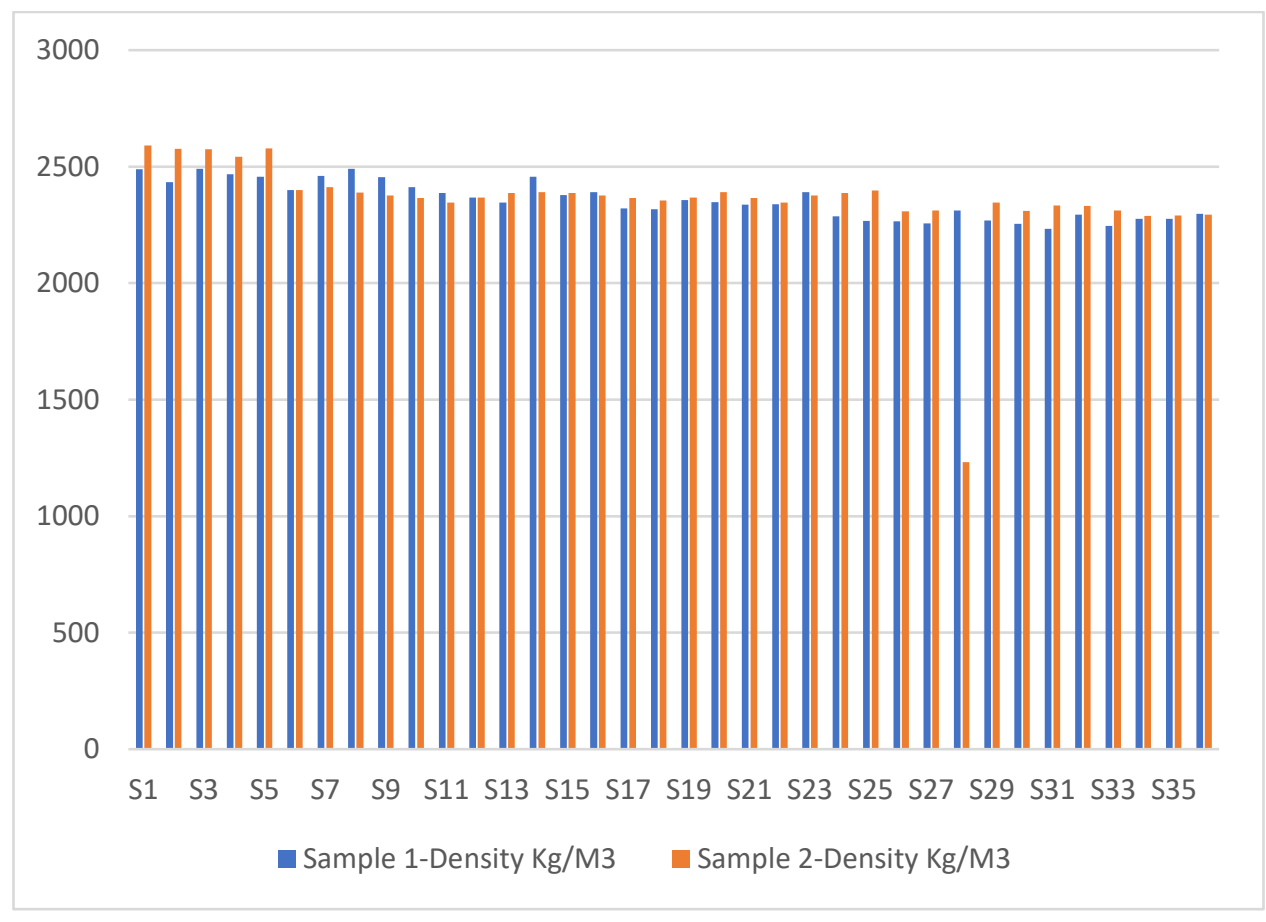

Figure 1: Density Comparison

In total, there were two main samples as one without rice husk ash and the other with rice husk ash. There were total of 36 sub-samples for both main samples. Results shows that for sample 1, the density $\mathrm{kg} / \mathrm{m} 3$ without rice husk ash was 2488 and with rice husk ash it was 2590. For 
sample 2, the density for excluded sample was 2434 and included sample was 2576. For sample 3 , the density for excluded sample was 2490 and included sample was 2574. For sample 3, the density for excluded sample was 2490 and included sample was 2574 . For sample 4, the density for excluded sample was 2467 and included sample was 2543. For sample 5, the density for excluded sample was 2456 and included sample was 2578. For sample 6, the density for excluded sample was 2400 and included sample was 2400 .For sample 7, the density for excluded sample was 2461 and included sample was 2412. For sample 8, the density for excluded sample was 2491 and included sample was 2389. For sample 9, the density for excluded sample was 2455 and included sample was 2376. For sample 10, the density for excluded sample was 2412 and included sample was 2365. For sample 11, the density for excluded sample was 2387 and included sample was 2345. Sample 12, the density for excluded sample was 2367 and included sample was 2367.For sample 13, the density for excluded sample was 2345 and included sample was 2387. For sample 14, the density for excluded sample was 2456 and included sample was 2390.For sample 15, the density for excluded sample was 2378 and included sample was 2387. For sample 16, the density for excluded sample was 2390 and included sample was 2376. For sample 17, the density for excluded sample was 2320 and included sample was 2365 . For sample 18, the density for excluded sample was 2318 and included sample was 2354 . For sample 19, the density for excluded sample was 2356 and included sample was 2367. Sample 20, the density for excluded sample was 2347 and included sample was 2390. For sample 21, the density for excluded sample was 2336 and included sample was 2365. For sample 22, the density for excluded sample was 2338 and included sample was 2345. Sample 23, the density for excluded sample was 2391 and included sample was 2377. For sample 24, the density for excluded sample was 2286 and included sample was 2387. For sample 25, the density for excluded sample was 2267 and included sample was 2398. For sample 26, the density for excluded sample was 2265 and included sample was 2309. Sample 27, the density for excluded sample was 2256 and included sample was 2312. Sample 28, the density for excluded sample was 2311 and included sample was 2132. For Sample 29, the density for excluded sample was 2269 and included sample was 2345 . For sample 30, the density for excluded sample was 2255 and included sample was 2310. Sample 31, the density for excluded sample was 2234 and included sample was 2333. For sample 32, the density for excluded sample was 2294 and included sample was 2332. For sample 33, the density for excluded sample was 2245 and included sample was 2311. For sample 34 , the density for excluded sample was 2276 and included sample was 2289. For sample 35, the density for excluded sample was 2278 and included sample was 2290 . For sample 36, the density for excluded sample was 2298 and included sample was 2294.

The Average density for excluded sample was 2355 and for included sample was 2354 which shows that they are almost same. The statistical difference was also insignificant (t-stat $=0.766$, $\mathrm{P}>$.05). 


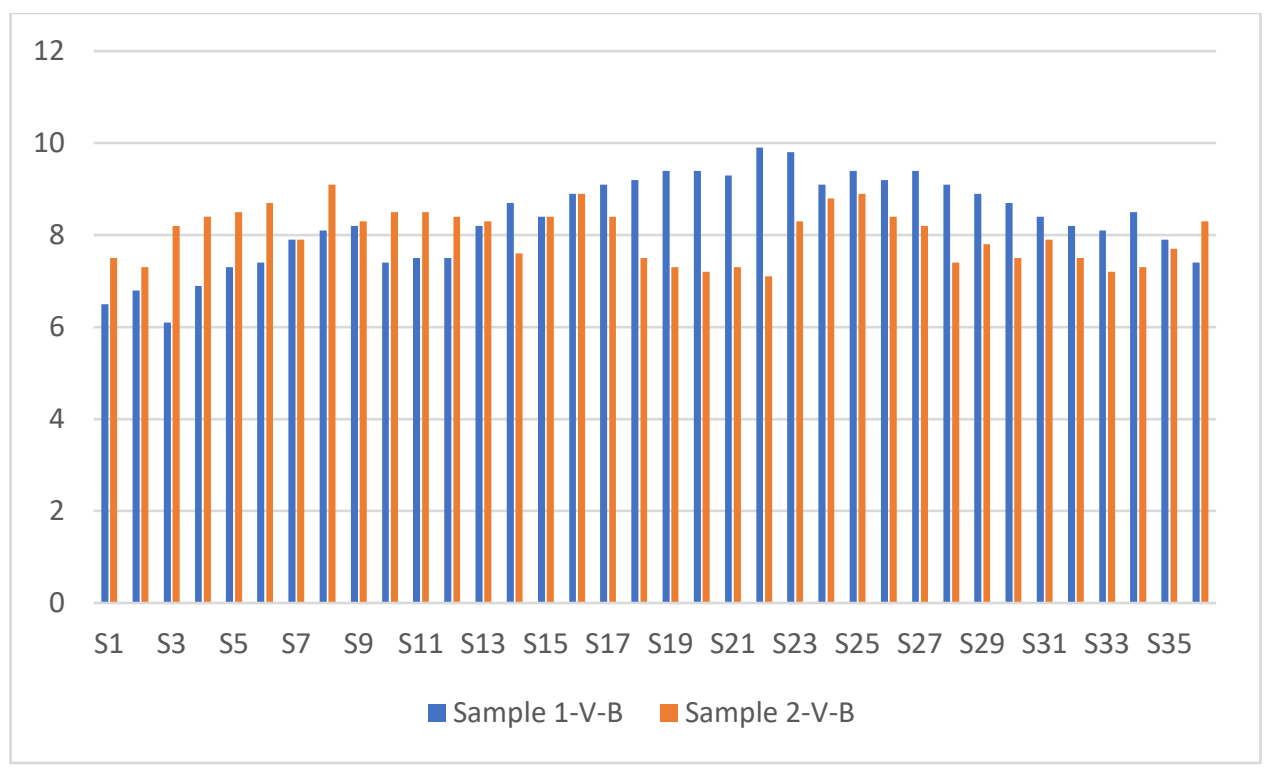

Figure 2: V-B Comparison

The results for the V-B shows that for sample 1, the VB for excluded sample was 6.5 and included sample was 7.5. For sample 2, the VB for excluded sample was 6.8 and included sample was 7.3. For sample 3, the VB for excluded sample was 6.1 and included sample was 8.2. For sample 4, the VB for excluded sample was 6.4 and included sample was 8.4. For sample 5, the VB for excluded sample was 7.3 and included sample was 8.5. or sample 6, the VB for excluded sample was 7.4 and included sample was 8.7. For sample 7, the VB for excluded sample was 7.9 and included sample was 7.9. For sample 8, the VB for excluded sample was 7.4 and included sample was 9.1. For sample 9, the VB for excluded sample was 8.2 and included sample was 8.3. For sample 10, the VB for excluded sample was 7.4 and included sample was 8.5. For sample 11, the VB for excluded sample was 7.5 and included sample was 8.5. For sample 12, the VB for excluded sample was 7.5 and included sample was 8.4. For sample 13, the VB for excluded sample was 8.2 and included sample was 8.3. For sample 14, the VB for excluded sample was 8.7 and included sample was 7.6. For sample 15, the VB for excluded sample was 8.4 and included sample was 8.4 . For sample 16 , the VB for excluded sample was 8.9 and included sample was 8.9. For sample 17, the VB for excluded sample was 9.1 and included sample was 8.4. For sample 18, the VB for excluded sample was 9.2 and included sample was 7.5. For sample 19, the VB for excluded sample was 9.4 and included sample was 7.3. For sample 20, the VB for excluded sample was 9.4 and included sample was 7.2. For sample 21, the VB for excluded sample was 9.3 and included sample was 7.3. For sample 22, the VB for excluded sample was 9.9 and included sample was 7.1. For sample 23, the VB for excluded sample was 9.8 and included sample was 8.3. For sample 24, the VB for excluded sample was 9.1 and included sample was 8.8 . For sample 25 , the VB for excluded sample was 9.4 and included sample was 8.9. For sample 26, the VB for excluded sample was 9.2 and included sample was 8.4. For sample 27, the VB for excluded sample was 9.4 and included sample was 8.2. For sample 28, the VB for excluded sample was 9.1 and included sample was 
7.4. For sample 29, the VB for excluded sample was 8.9 and included sample was 7.8. For sample 30, the VB for excluded sample was 8.7 and included sample was 7.5. For sample 31, the VB for excluded sample was 8.4 and included sample was 7.9. For sample 32, the VB for excluded sample was 8.2 and included sample was 7.5. For sample 33, the VB for excluded sample was 8.1 and included sample was 7.2. For sample 34, the VB for excluded sample was 8.5 and included sample was 7.3. For sample 35, the VB for excluded sample was 7.9 and included sample was 7.7. For sample 36, the VB for excluded sample was 7.4 and included sample was 8.3. The average VB for excluded sample was 8.34 and for included sample was 8.01. Statistically, the difference was insignificant $(\mathrm{t}$-stat=1.431, $\mathrm{P}>.05)$.

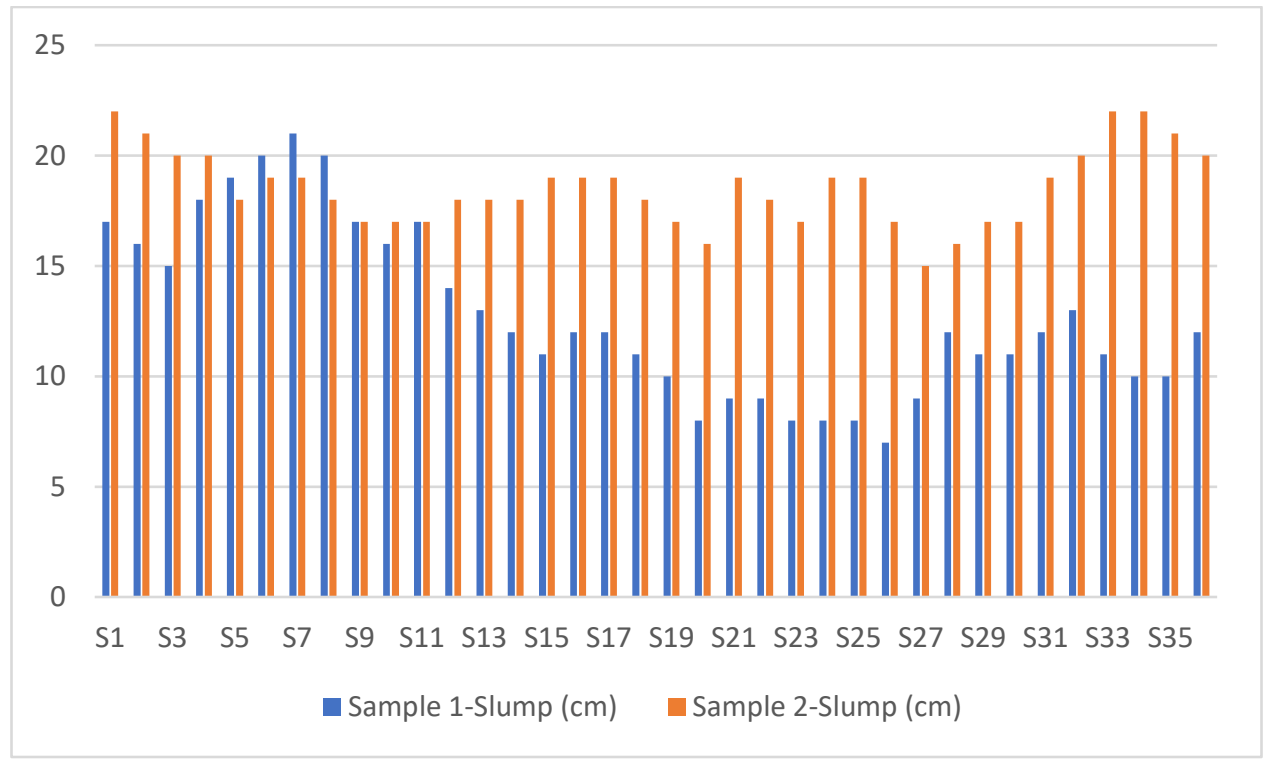

Figure 3: Slump Comparison

The slump test for both samples are as follows. For sample 1, the slump score for excluded sample was 17 and included sample was 22. For sample 2, the slump score for excluded sample was 16 and included sample was 21. For sample 3, the slump score for excluded sample was 15 and included sample was 20. For sample 4, the slump score for excluded sample was 18 and included sample was 20. or sample 5, the slump score for excluded sample was 19 and included sample was 18. For sample 6, the slump score for excluded sample was 20 and included sample was 19. For sample 7, the slump score for excluded sample was 21 and included sample was 19. For sample 8, the slump score for excluded sample was 20 and included sample was 18 . For sample 9, the slump score for excluded sample was 17 and included sample was 17. For sample 10, the slump score for excluded sample was 16 and included sample was 17. For sample 11, the slump score for excluded sample was 17 and included sample was 17. For sample 12, the slump score for excluded sample was 14 and included sample was 18. For sample 13, the slump score for excluded sample was 13 and included sample was 18. Sample 14, the slump score for excluded sample was 12 and included sample was 18. For sample 15, the slump score for excluded sample was 11 and included sample was 19. Sample 16, the slump score for excluded 
sample was 12 and included sample was 19. Sample 17, the slump score for excluded sample was 12 cand included sample was 19 . For sample 18, the slump score for excluded sample was 11 and included sample was 18 . For sample 19, the slump score for excluded sample was 10 and included sample was 17. For sample 20, the slump score for excluded sample was 8 and included sample was 16. For sample 21, the slump score for excluded sample was 9 and included sample was 19. For sample 22, the slump score for excluded sample was 9 and included sample was 18. For sample 23, the slump score for excluded sample was 8 and included sample was 17 . For sample 24, the slump score for excluded sample was 8 and included sample was 19 . For sample 25 , the slump score for excluded sample was 8 and included sample was 19 . For sample 26, the slump score for excluded sample was 7 and included sample was 17. For sample 27, the slump score for excluded sample was 9 and included sample was 15 . For sample 28, the slump score for excluded sample was 12 and included sample was 16. For sample 29, the slump score for excluded sample was 11 and included sample was 17. For sample 30, the slump score for excluded sample was 11 and included sample was 17. Sample 31, the slump score for excluded sample was 12 and included sample was 19. For sample 32, the slump score for excluded sample was 13 and included sample was 20. For sample 33, the slump score for excluded sample was 11 and included sample was 22. Sample 34, the slump score for excluded sample was 10 and included sample was 22. For sample 35, the slump score for excluded sample was 10 and included sample was 21. Lastly, for sample 36, the slump score for excluded sample was 12 and included sample was 20. The average for slump test for excluded sample was 12.75 and for included sample was 18.56 . The difference was statistically significant ( $\mathrm{t}$-stat=2.455, $\mathrm{P}<.05)$.

\section{Compressive Strength}

The period of 4 week is used for testing the compressive strength test. Results are as follows.

Table 2: Compressive Strength Comparison

\begin{tabular}{|l|c|c|}
\hline No. & $\begin{array}{c}\text { Sample 1- Compressive Strength- } \\
\text { Without Rice Husk Ash }\end{array}$ & $\begin{array}{c}\text { Sample 2-Compressive Strength- } \\
\text { With Rice Husk Ash }\end{array}$ \\
\hline S1 & 19.56 & 18.56 \\
\hline S2 & 18.45 & 19.32 \\
\hline S3 & 19.43 & 19.43 \\
\hline S4 & 17.9 & 18.56 \\
\hline S5 & 19.4 & 19.43 \\
\hline S6 & 20.45 & 19.32 \\
\hline S7 & 21.34 & 19.04 \\
\hline S8 & 21.45 & 19.41 \\
\hline S9 & 22.34 & 19.44 \\
\hline S10 & 23.45 & 18.46 \\
\hline S11 & 23.76 & 18.73 \\
\hline S12 & 21.23 & 18.68 \\
\hline S13 & 20.55 & 18.33 \\
\hline S14 & 22.87 & 18.49 \\
\hline
\end{tabular}




\begin{tabular}{|l|c|c|} 
& 21.9 & 18.72 \\
\hline S16 & 18.67 & 18.43 \\
\hline S17 & 19.03 & 20.43 \\
\hline S18 & 19.56 & 20.99 \\
\hline S19 & 18.55 & 20.45 \\
\hline S20 & 17.34 & 21.37 \\
\hline S21 & 18.65 & 21.56 \\
\hline S22 & 19.34 & 21.66 \\
\hline S23 & 18.77 & 21.33 \\
\hline S24 & 17.33 & 21.89 \\
\hline S25 & 18.43 & 21.55 \\
\hline S26 & 17.45 & 20.66 \\
\hline S27 & 19.43 & 20.63 \\
\hline S28 & 19.34 & 20.98 \\
\hline S29 & 20.45 & 20.55 \\
\hline S30 & 21.55 & 20.77 \\
\hline S31 & 21.67 & 20.45 \\
\hline S32 & 21.52 & 20.66 \\
\hline S33 & 21.9 & 20.53 \\
\hline S34 & 23.34 & 20.66 \\
\hline S35 & 23.56 & 20.43 \\
\hline S36 & 24.32 & 20.56 \\
\hline Average & $\mathbf{2 0 . 4 0}$ & $\mathbf{2 0 . 0 1}$ \\
\hline & & \\
\hline & & \\
\hline
\end{tabular}

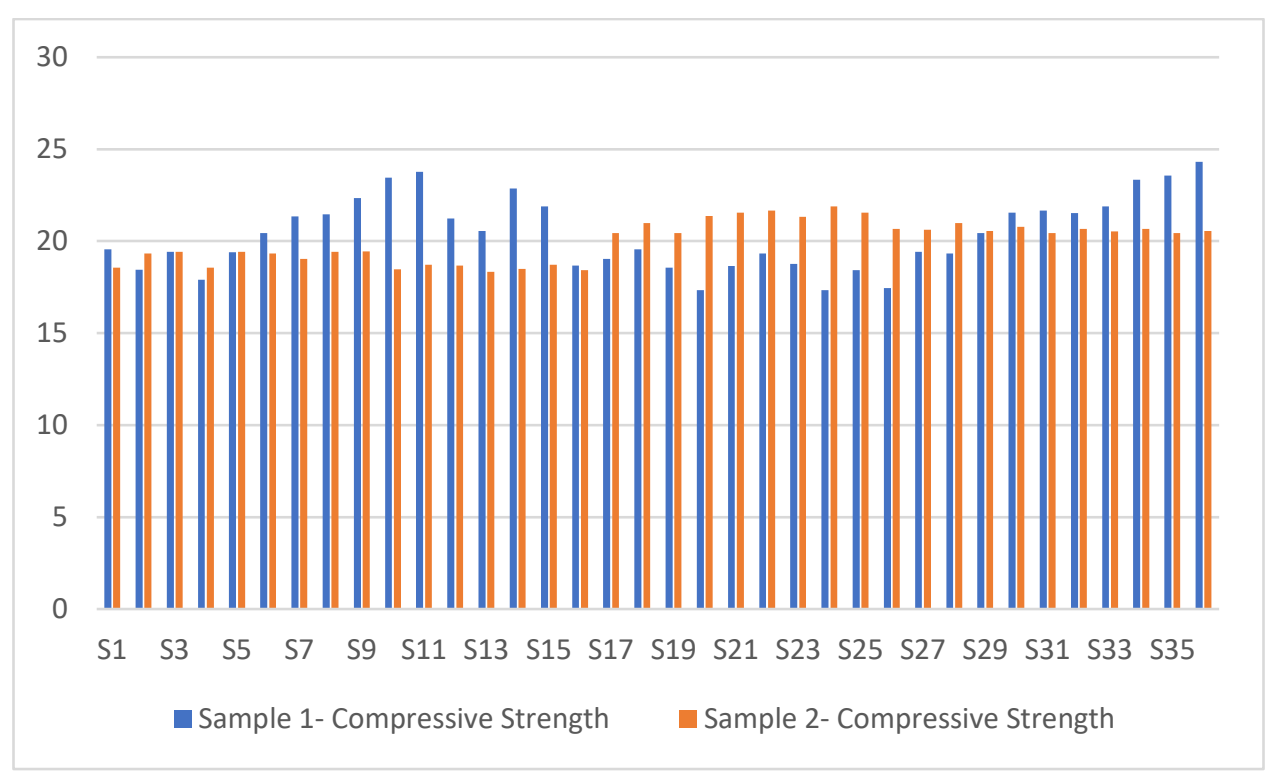

Figure 4: Compressive Strength 
The results for compressive strength are as follows. For sample 1, the score for compressive strength for excluded sample was 19.56, and for included sample was 18.56. For sample 2, the score for compressive strength for excluded sample was 18.45 and for included sample was 19.32. For sample 3, the score for compressive strength for excluded sample was 19.43 and for included sample was 19.43. For sample 4, the score for compressive strength for excluded sample was 17.90 and for included sample was 18.56.For sample 5, the score for compressive strength for excluded sample was 19.40 and for included sample was 19.43.For sample 6, the score for compressive strength for excluded sample was 20.45 and for included sample was 19.32. For sample 7, the score for compressive strength for excluded sample was 21.34 and for included sample was 19.04. For sample 8, the score for compressive strength for excluded sample was 21.45 and for included sample was 19.41. For sample 9, the score for compressive strength for excluded sample was 22.34 and for included sample was 19.44. For sample 10, the score for compressive strength for excluded sample was 23.45 and for included sample was 18.46. For sample 11, the score for compressive strength for excluded sample was 23.76 and for included sample was 18.73. For sample 12, the score for compressive strength for excluded sample was 21.23 and for included sample was 18.68. For sample 13, the score for compressive strength for excluded sample was 20.55 and for included sample was 18.33. For sample 14, the score for compressive strength for excluded sample was 22.87 and for included sample was 18.49. For sample 15, the score for compressive strength for excluded sample was 21.9 and for included sample was 18.72. For sample 16, the score for compressive strength for excluded sample was 18.67 and for included sample was 18.43. For sample 17, the score for compressive strength for excluded sample was 19.03 and for included sample was 20.43. For sample 18, the score for compressive strength for excluded sample was 19.56 and for included sample was 20.99. For sample 19, the score for compressive strength for excluded sample was 18.55 and for included sample was 20.45. For sample 20, the score for compressive strength for excluded sample was 17.34 and for included sample was 21.37. For sample 21, the score for compressive strength for excluded sample was 18.65 and for included sample was 21.56. For sample 22, the score for compressive strength for excluded sample was 19.34 and for included sample was 21.66. For sample 23, the score for compressive strength for excluded sample was 18.77 and for included sample was 21.33. For sample 24, the score for compressive strength for excluded sample was 17.33 and for included sample was 21.89. For sample 25, the score for compressive strength for excluded sample was 18.43 and for included sample was 21.55. For sample 26, the score for compressive strength for excluded sample was 17.45 and for included sample was 20.66. For sample 27, the score for compressive strength for excluded sample was 19.43 and for included sample was 20.63. For sample 28, the score for compressive strength for excluded sample was 19.34 and for included sample was 20.98. For sample 29, the score for compressive strength for excluded sample was 20.45 and for included sample was 20.55. For sample 30, the score for compressive strength for excluded sample was 21.55 and for included sample was 20.77. For sample 31, the score for compressive strength for excluded sample was 21.67 and for included sample was 20.45. For sample 32, the score for compressive strength for excluded 
sample was 21.52 and for included sample was 20.66. For sample 33, the score for compressive strength for excluded sample was 21.9 and for included sample was 20.53. For sample 34, the score for compressive strength for excluded sample was 23.34 and for included sample was 20.66. For sample 35, the score for compressive strength for excluded sample was 23.56 and for included sample was 20.43. For sample 36, the score for compressive strength for excluded sample was 24.32 and for included sample was 20.01. On average, the excluded sample showed better compressive strength compared to the included sample. The results were statistically insignificant (t-value $=1.13, \mathrm{P}>.05)$.

\section{Flexural Tensile Strength}

Flexural tensile strength test is conducted on steel fiber reinforced concrete with and without rice husks ash.

Table 3: Flexural Strength Comparison

\begin{tabular}{|c|c|c|}
\hline No. & $\begin{array}{c}\text { Sample 1-Flexural } \\
\text { Strength }\end{array}$ & $\begin{array}{c}\text { Sample 2-Flexural } \\
\text { Strength } \\
\end{array}$ \\
\hline $\mathrm{S} 1$ & 8.56 & 8.56 \\
\hline S2 & 8.65 & 8.52 \\
\hline S3 & 8.65 & 8.65 \\
\hline S4 & 7.9 & 8.56 \\
\hline S5 & 8.6 & 8.65 \\
\hline S6 & 9.65 & 8.52 \\
\hline S7 & 9.56 & 8.06 \\
\hline S8 & 9.65 & 8.61 \\
\hline S9 & 9.56 & 8.66 \\
\hline $\mathrm{S} 10$ & 9.65 & 8.66 \\
\hline S11 & 9.76 & 8.75 \\
\hline $\mathrm{S} 12$ & 9.9 & 8.68 \\
\hline S13 & 9.55 & 8.55 \\
\hline S14 & 9.87 & 8.69 \\
\hline S15 & 9.9 & 8.72 \\
\hline S16 & 8.67 & 8.65 \\
\hline S17 & 8.05 & 9.65 \\
\hline $\mathrm{S} 18$ & 8.56 & 9.99 \\
\hline S19 & 8.55 & 9.65 \\
\hline S20 & 7.56 & 9.57 \\
\hline S21 & 8.65 & 9.56 \\
\hline S22 & 8.56 & 9.66 \\
\hline $\mathrm{S} 23$ & 8.77 & 9.55 \\
\hline S24 & 7.55 & 9.89 \\
\hline S25 & 8.65 & 9.55 \\
\hline S26 & 7.65 & 9.66 \\
\hline
\end{tabular}




\begin{tabular}{|c|c|c|} 
S27 & 8.65 & 9.65 \\
\hline S28 & 8.56 & 9.98 \\
\hline S29 & 9.65 & 9.55 \\
\hline S30 & 9.55 & 9.77 \\
\hline S31 & 9.67 & 9.65 \\
\hline S32 & 9.52 & 9.66 \\
\hline S33 & 9.9 & 9.55 \\
\hline S34 & 9.56 & 9.66 \\
\hline S35 & 9.56 & 9.65 \\
\hline S36 & 9.52 & 9.56 \\
\hline Average & $\mathbf{9 . 0 2}$ & $\mathbf{9 . 1 9}$ \\
\hline
\end{tabular}

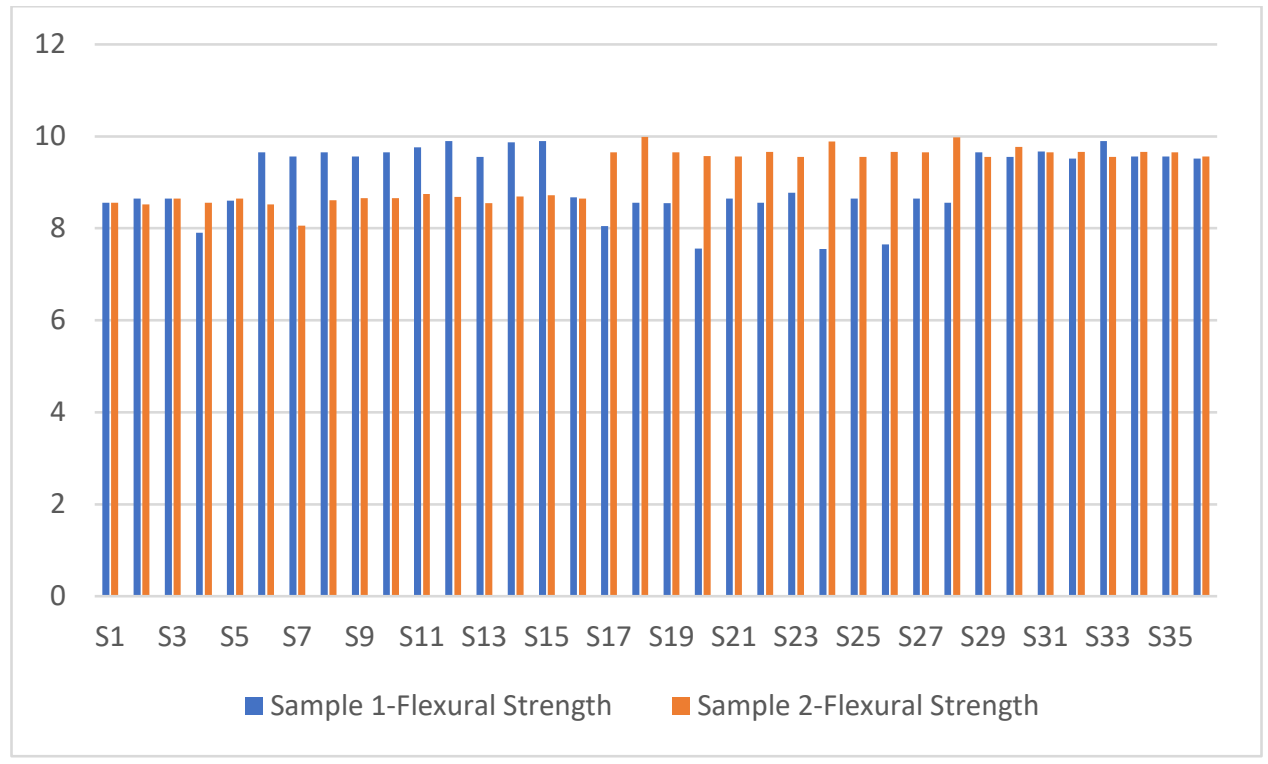

Figure 5: Flexural Strength Comparison

The results for flexural strength test are given in the table above. For sample 1, the flexural strength for excluded sample was 8.56 and for included sample was 8.56. For sample 2, the flexural strength for excluded sample was 8.65 and for included sample was 8.52. For sample 3, the flexural strength for excluded sample was 8.65 and for included sample was 8.65. For sample 4, the flexural strength for excluded sample was 7.9 and for included sample was 8.56. For sample 5, the flexural strength for excluded sample was 8.6 and for included sample was 8.65. For sample 6, the flexural strength for excluded sample was 9.65 and for included sample was 8.52. For sample 7, the flexural strength for excluded sample was 9.56 and for included sample was 8.06. For sample 8, the flexural strength for excluded sample was 9.65 and for included sample was 8.61. For sample 9, the flexural strength for excluded sample was 9.65 and for included sample was 8.66. For sample 10, the flexural strength for excluded sample was 9.65 and for included sample was 8.66. For sample 11, the flexural strength for excluded sample was 9.76 and for included sample was 8.75. For sample 12, the flexural strength for excluded sample was 
9.9 and for included sample was 8.68. For sample 13, the flexural strength for excluded sample was 9.55 and for included sample was 8.55 . For sample 14, the flexural strength for excluded sample was 9.87 and for included sample was 8.69. For sample 15, the flexural strength for excluded sample was 9.9 and for included sample was 8.72. For sample 16, the flexural strength for excluded sample was 8.67 and for included sample was 8.65. For sample 17, the flexural strength for excluded sample was 8.05 and for included sample was 9.65. For sample 18, the flexural strength for excluded sample was 8.56 and for included sample was 9.99. For sample 19, the flexural strength for excluded sample was 8.55 and for included sample was 9.56. For sample 20, the flexural strength for excluded sample was 7.56 and for included sample was 9.57. For sample 21, the flexural strength for excluded sample was 8.65 and for included sample was 9.56. For sample 22, the flexural strength for excluded sample was 8.56 and for included sample was 9.66. For sample 23, the flexural strength for excluded sample was 8.77 and for included sample was 9.55. or sample 24, the flexural strength for excluded sample was 7.55 and for included sample was 9.89. For sample 25, the flexural strength for excluded sample was 8.65 and for included sample was 9.55 . For sample 26, the flexural strength for excluded sample was 8.56 and for included sample was 9.66. For sample 27, the flexural strength for excluded sample was 7.65 and for included sample was 9.65. For sample 28, the flexural strength for excluded sample was 8.56 and for included sample was 9.98. For sample 29, the flexural strength for excluded sample was 9.65 and for included sample was 9.55. For sample 30, the flexural strength for excluded sample was 9.55 and for included sample was 9.77. For sample 31, the flexural strength for excluded sample was 9.67 and for included sample was 9.65. For sample 32, the flexural strength for excluded sample was 9.52 and for included sample was 9.66. For sample 33, the flexural strength for excluded sample was 9.9 and for included sample was 9.66. For sample 34, the flexural strength for excluded sample was 9.56 and for included sample was 9.65. For sample 35, the flexural strength for excluded sample was 9.56 and for included sample was 9.56. For sample 36, the flexural strength for excluded sample was 9.52 and for included sample was 9.19. The average for excluded sample was 9.02 and for included sample was 9.19. The difference was statistically insignificant ( $\mathrm{t}$-stat=1.45, $\mathrm{P}>.05)$.

\section{CONCLUSION}

The study was experimental in nature and conducted to test the effects of addition of rice husk ash in concrete. It divided two sample groups one with the rice husk ash and the other one without rice husk ash. Different tests were performed including compressive strength, flexural strength, and splitting tensile strength. The results showed that both samples did not have significant differences and most results were very similar. This led to the conclusion that addition of rice husk ash in concrete bring no significant change.

\section{References}

Eren, O., \& Celik, T. (1997). Effect of silica fume and steel fibers on some properties of highstrength concrete. Construction Building Material, 11, 373-82 
Mohammadi, Y., Singh, S.P., \& Kaushik, S.K. (2006). Properties of steel fibrous concrete containing mixed fibers in fresh and hardened state. Construction Building Material, Doi:10.1016/j.conbuildmat.2006.12.004

Yazici, S., Inan, G., \& Tabak, V. (2007). Effect of aspect ratio and volume fraction of steel fiber on the mechanical properties of SFRC. Construction Building Material, 21(3), 1250-3.

Zollo, R.F. (1997). Fiber-reinforced concrete: an overview after 30 years of development. Cement Concrete Composition, 19, 107-22 